\title{
SOLUSI SISTEM PERSAMAAN DIFERENSIAL LINEAR FRACTIONAL DENGAN TURUNAN TIPE JUMARIE
}

\author{
RAMADHAL NOFRINA PUTRI, RADHIATUL HUSNA* \\ Program Studi S1 Matematika, \\ Fakultas Matematika dan Ilmu Pengetahuan Alam, Universitas Andalas, \\ Kampus UNAND Limau Manis Padang, Indonesia. \\ email : ramadhal.rina@gmail.com,radhiatulhusna@sci.unand.ac.id
}

Diterima 16 Juni 2020 Direvisi 22 Juni 2020 Dipublikasikan 13 Juli 2020

\begin{abstract}
Abstrak. Dalam makalah ini ditentukan solusi sistem persamaan diferensial fractional linier orde dengan turunan tipe Jumarie. Bentuk umum solusi diberikan dalam beberapa teorema. Beberapa contoh diberikan untuk mengilustrasikan keberlakuan teorema.
\end{abstract}

Kata Kunci: Persamaan Diferensial Fraksional, Jumarie, Fungsi Mittag-Leffler

\section{Pendahuluan}

Suatu persamaan diferensial biasa adalah suatu persamaan yang memuat suatu fungsi dan turunan-turunan biasa dari fungsi tersebut. Sistem persamaan diferensial biasa adalah kumpulan dari beberapa persamaan diferensial biasa.

Seiring dengan perkembangan, persamaan diferensial biasa berkembang menjadi persamaan diferensial fractional. Selanjutnya sistem persamaan diferensial biasa juga berkembang menjadi sistem persamaan diferensial fractional. Dalam makalah ini akan dibahas mengenai solusi sistem persamaan diferensial fractional berikut ([2] dan [5]):

$$
\begin{aligned}
& D^{\alpha} x(t)=a x+b y \\
& D^{\alpha} y(t)=c x+d y
\end{aligned}
$$

dimana $a, b, c$ dan $d$ adalah konstanta dan $D^{\alpha}$ adalah turunan fractional tipe Jumarie orde $\alpha$ dengan $0<\alpha<1$ yang didefinisikan sebagai berikut [2]:

$$
D^{\alpha} f(t)=\frac{1}{\Gamma(1-\alpha)} \frac{d}{d t} \int_{0}^{t}(t-\tau)^{-\alpha}(f(\tau)-f(0)) d \tau
$$

dengan $\Gamma$ adalah fungsi Gamma.

${ }^{*}$ penulis korespondensi 
208 Ramadhal Nofrina Putri, Radhiatul Husna

\section{Landasan Teori}

\subsection{Fungsi Gamma, Fungsi Beta dan Fungsi Mittag-Leffler}

Definisi 2.1. [7] Fungsi Gamma dinyatakan sebagai $\Gamma(n)$, didefinisikan sebagai berikut:

$$
\Gamma(n)=\int_{0}^{\infty} x^{n-1} e^{-x} d x, \quad n>0 .
$$

Definisi 2.2. [5] Fungsi Beta didefinisikan sebagai berikut :

$$
B(p, q)=\int_{0}^{1} x^{p-1}(1-x)^{q-1} d x, x \in \mathbb{R} \text { dan } p, q \in \mathbb{C},
$$

dimana $\operatorname{Re}(p)>0$ dan $\operatorname{Re}(q)>0$.

Definisi 2.3. [2] Fungsi Mittag-Leffler satu parameter $E_{\alpha}$ didefinisikan sebagai berikut:

$$
E_{\alpha}(z)=\sum_{k=0}^{\infty} \frac{z^{k}}{\Gamma(1+\alpha k)}, \quad z \in \mathbb{C}, \quad \operatorname{Re}(\alpha)>0 .
$$

\subsection{Turunan Fractional Tipe Jumarie}

Definisi 2.4. [1] Turunan fraksional Jumarie orde $\alpha$ dari fungsi $f(t), t>0$ dengan $0<\alpha<1$, dinotasikan dengan $D^{\alpha} f(t)$, didefinisikan sebagai berikut:

$$
D^{\alpha} f(t)=\frac{1}{\Gamma(1-\alpha)} \frac{d}{d t} \int_{0}^{t}(t-\tau)^{-\alpha}(f(\tau)-f(0)) d \tau .
$$

Beberapa sifat dari turunan fractional Jumarie diberikan berikut ini [4]:

(1) $D^{\alpha}[\lambda f(t)]=\lambda D^{\alpha}[f(t)]$

(2) $D^{\alpha}[f(t)+g(t)]=D^{\alpha}[f(t)]+D^{\alpha}[g(t)]$

(3) $D^{\alpha}[f(t) g(t)]=\left[D^{\alpha} f(t)\right] g(t)+f(t)\left[D^{\alpha} g(t)\right]$.

Dengan menggunakan persamaan (2.3), diperoleh turunan dari beberapa fungsi berikut.

(1) $D^{\alpha}(c)=0$.

(2) $D^{\alpha}\left(t^{n \alpha}\right)=\frac{\Gamma(n \alpha+1)}{\Gamma(\alpha(n-1)+1)} t^{(\alpha(n-1)}$.

(3) $D^{\alpha}\left(E_{\alpha}\left(\lambda t^{\alpha}\right)\right)=\lambda E_{\alpha}\left(\lambda t^{\alpha}\right.$.

(4) $D^{\alpha}\left(\cos _{\alpha}\left(t^{\alpha}\right)\right)=-\sin _{\alpha}\left(t^{\alpha}\right)$.

(5) $D^{\alpha}\left(\cos _{\alpha}\left(\lambda t^{\alpha}\right)\right)=-\lambda \sin _{\alpha}\left(\lambda t^{\alpha}\right)$. 


\section{Pembahasan}

Berikut ini diperlihatkan bagaimana turunan Jumarie digunakan untuk menyelesaikan beberapa sistem persamaan diferensial fraksional linier orde $\alpha$.

$$
\begin{aligned}
& D^{\alpha} x=a x+b y, \\
& D^{\alpha} y=c x+d y .
\end{aligned}
$$

$a, b, c$ dan $d$ adalah konstanta dan $D^{\alpha}$ adalah turunan fractional tipe Jumarie orde $\alpha$ dengan $0<\alpha<1$.

Dari persamaan (3.1) diperoleh:

$$
\begin{aligned}
\left(D^{\alpha}-a\right) x-b y & =0, \\
-c x+\left(D^{\alpha}-d\right) y & =0 .
\end{aligned}
$$

Penggunaan (3.2) dan pengoperasian (3.3) dengan $\left(D^{\alpha}-a\right)$ menghasilkan:

$$
\begin{aligned}
-c\left(D^{\alpha}-a\right) x+\left(D^{\alpha}-a\right)\left(D^{\alpha}-d\right) y & =0, \\
-c b y+\left(D^{\alpha}-a\right)\left(D^{\alpha}-d\right) y & =0, \\
D^{2 \alpha} y-(a+d)\left(D^{\alpha} y\right)+(a d-b c) y & =0 .
\end{aligned}
$$

Persamaan (3.4) adalah persamaan diferensial fractional linear orde $2 \alpha$.

Teorema 3.1. [2] Jika $(a+d)^{2}-4(a d-b c)>0$, maka solusi dari (3.1) adalah

$$
\begin{aligned}
& x=A_{1} E_{\alpha}\left(\lambda_{1} t^{\alpha}\right)+B_{1} E_{\alpha}\left(\lambda_{2} t^{\alpha}\right) \\
& y=A_{2} E_{\alpha}\left(\lambda_{1} t^{\alpha}\right)+B_{2} E_{\alpha}\left(\lambda_{2} t^{\alpha}\right)
\end{aligned}
$$

dimana $A_{2}$ dan $B_{2}$ adalah konstanta sebarang dan $A_{1}=\frac{A_{2}\left(\lambda_{1}-d\right)}{c}$ dan $B_{1}=$ $\frac{B_{2}\left(\lambda_{2}-d\right)}{c}$.

Bukti. Turunan fractional ke $\alpha$ dari persamaan kedua (3.5) adalah

$$
D^{\alpha} y=A_{2} \lambda_{1} E_{\alpha}\left(\lambda_{1} t^{\alpha}\right)+B_{2} \lambda_{2} E_{\alpha}\left(\lambda_{2} t^{\alpha}\right) .
$$

Pengurangan (3.6) dengan $\lambda_{1} y$ diperoleh sebagai berikut:

$$
\begin{aligned}
D^{\alpha} y-\lambda_{1} y & =A_{2} \lambda_{1} E_{\alpha}\left(\lambda_{1} t^{\alpha}\right)+B_{2} \lambda_{2} E_{\alpha}\left(\lambda_{2} t^{\alpha}\right)-\lambda_{1} y, \\
& =A_{2} \lambda_{1} E_{\alpha}\left(\lambda_{1} t^{\alpha}\right)+B_{2} \lambda_{2} E_{\alpha}\left(\lambda_{2} t^{\alpha}\right)-\lambda_{1}\left(A_{2} E_{\alpha}\left(\lambda_{1} t^{\alpha}\right)+B_{2} E_{\alpha}\left(\lambda_{2} t^{\alpha}\right)\right), \\
& =B_{2}\left(\lambda_{2}-\lambda_{1}\right) E_{\alpha}\left(\lambda_{2} t^{\alpha}\right) .
\end{aligned}
$$

Turunan ke $\alpha$ dari (3.7) adalah:

$$
\begin{aligned}
D^{\alpha}\left(D^{\alpha}-\lambda_{1} y\right) & =D^{\alpha}\left(B_{2}\left(\lambda_{2}-\lambda_{1}\right) E_{\alpha}\left(\lambda_{2} t^{\alpha}\right)\right), \\
D^{2 \alpha} y-\lambda_{1} D^{\alpha} y & =B_{2} \lambda_{2}\left(\lambda_{2}-\lambda_{1}\right) E_{\alpha}\left(\lambda_{2} t^{\alpha}\right) .
\end{aligned}
$$

Dengan mensubstitusi (3.7) ke persamaan (3.9) diperoleh:

$$
\begin{aligned}
D^{2 \alpha} y-\lambda_{1} D^{\alpha} y & =\lambda_{2}\left(D^{\alpha} y-\lambda_{1} y\right), \\
& =\lambda_{2} D^{\alpha} y-\lambda_{1} \lambda_{2} y, \\
D^{2 \alpha} y-(a+d) D^{\alpha} y+(a d-b c) y & =0 .
\end{aligned}
$$


Persamaan (3.10) menunjukkan bahwa persamaan kedua (3.5) adalah solusi dari persamaan (3.4). Untuk mendapatkan solusi $x$ dari persamaan (3.1), substitusi persamaan kedua dari (3.5) ke persamaan kedua dari (3.1), yaitu

$$
\begin{aligned}
D^{\alpha} y & =c x+d y \\
D^{\alpha}\left[A_{2} E_{\alpha}\left(\lambda_{1} t^{\alpha}\right)+B_{2} E_{\alpha}\left(\lambda_{2} t^{\alpha}\right)\right] & =c x+d\left(A E_{\alpha}\left(\lambda_{1} t^{\alpha}\right)+B_{2} E_{\alpha}\left(\lambda_{2} t^{\alpha}\right)\right), \\
c x & =\left[A_{2} \lambda_{1} E_{\alpha}\left(\lambda_{1} t^{\alpha}\right)+B_{2} \lambda_{2} E_{\alpha}\left(\lambda_{2} t^{\alpha}\right)\right]-d\left(A_{2} E_{\alpha}\left(\lambda_{1} t^{\alpha}\right)+B_{2} E_{\alpha}\left(\lambda_{2} t^{\alpha}\right)\right), \\
c x & =A_{2}\left(\lambda_{1}-d\right) E_{\alpha}\left(\lambda_{1} t^{\alpha}\right)+B_{2}\left(\lambda_{2}-d\right) E_{\alpha}\left(\lambda_{2} t^{\alpha}\right) .
\end{aligned}
$$

atau dapat ditulis:

$$
\begin{aligned}
x & =\frac{1}{c}\left[A_{2}\left(\lambda_{1}-d\right) E_{\alpha}\left(\lambda_{1} t^{\alpha}\right)+B_{2}\left(\lambda_{2}-d\right) E_{\alpha}\left(\lambda_{2} t^{\alpha}\right)\right], \\
& =A_{1} E_{\alpha}\left(\lambda_{1} t^{\alpha}\right)+B_{1} E_{\alpha}\left(\lambda_{2} t^{\alpha}\right), \text { dimana } \\
A_{1} & =\frac{A_{2}\left(\lambda_{1}-d\right)}{c} ; \quad B_{1}=\frac{B_{2}\left(\lambda_{2}-d\right)}{c} .
\end{aligned}
$$

Teorema 3.2. [2] Jika $(a+d)^{2}-4(a d-b c)=0$, maka solusi dari (3.1) adalah:

$$
\begin{aligned}
& x=\left(A_{1} t^{\alpha}+A_{2}\right) E_{\alpha}\left(\lambda t^{\alpha}\right), \\
& y=\left(B_{1} t^{\alpha}+B_{2}\right) E_{\alpha}\left(\lambda t^{\alpha}\right) .
\end{aligned}
$$

dimana $B_{1}$ dan $B_{2}$ adalah konstanta sebarang dan $A_{1}=\frac{(\lambda-d) B_{1}}{c}, A_{2}=$ $\frac{\Gamma(1+\alpha) B_{1}+(\lambda-d) B_{2}}{c}$.

Bukti. Turunan fractional ke $\alpha$ dari persamaan kedua (3.11) adalah:

$$
D^{\alpha} y=\Gamma(1+\alpha) B_{1} E_{\alpha}\left(\lambda t^{\alpha}\right)+\left(B_{1} t^{\alpha}+B_{2}\right) \lambda E_{\alpha}\left(\lambda t^{\alpha}\right) .
$$

Pengurangan (3.12) dengan $\lambda y$, memberikan:

$$
\begin{aligned}
D^{\alpha} y-\lambda y= & \Gamma(1+\alpha) B_{1} E_{\alpha}\left(\lambda t^{\alpha}\right)+\left(B_{1} t^{\alpha}+B_{2}\right) \lambda E_{\alpha}\left(\lambda t^{\alpha}\right) \\
& -\lambda\left(\left(B_{1} t^{\alpha}+B_{2}\right) E_{\alpha}\left(\lambda t^{\alpha}\right)\right), \\
= & \Gamma(1+\alpha) B_{1} E_{\alpha}\left(\lambda t^{\alpha}\right) .
\end{aligned}
$$

Turunan ke $\alpha$ dari (3.13) adalah:

$$
\begin{aligned}
D^{\alpha}\left(D^{\alpha} y-\lambda y\right) & =D^{\alpha}\left(\Gamma(1+\alpha) B_{1} E_{\alpha}\left(\lambda t^{\alpha}\right)\right), \\
D^{2 \alpha} y-\lambda D^{\alpha} y & =\lambda \Gamma(1+\alpha) B_{1} E_{\alpha}\left(\lambda t^{\alpha}\right), \\
& =\lambda\left(D^{\alpha} y-\lambda y\right), \\
D^{2 \alpha} y-\lambda D^{\alpha} y & =\lambda D^{\alpha} y-\lambda^{2} y, \\
D^{2 \alpha} y-2 \lambda D^{\alpha} y+\lambda^{2} y & =0, \\
D^{2 \alpha} y-(a+d) D^{\alpha} y+\frac{1}{4}(a+d)^{2} y & =0 .
\end{aligned}
$$

Persamaan (3.14) menunjukkan bahwa persamaan kedua (3.11) adalah solusi dari persamaan (3.4). Untuk mendapatkan solusi $x$ dari persamaan (3.1), substitusi per- 
samaan kedua dari (3.11) ke persamaan kedua dari (3.1), menghasilkan:

$$
\begin{gathered}
D^{\alpha} y=c x+d y, \\
D^{\alpha}\left[\left(B_{1} t^{\alpha}+B_{2}\right) E_{\alpha}\left(\lambda t^{\alpha}\right)\right]=c x+d\left[\left(B_{1} t^{\alpha}+B_{2}\right) E_{\alpha}\left(\lambda t^{\alpha}\right)\right], \\
\Gamma(1+\alpha) B_{1} E_{\alpha}\left(\lambda t^{\alpha}\right)+\left(B_{1} t^{\alpha}+B_{2}\right) \lambda E_{\alpha}\left(\lambda t^{\alpha}\right)=c x+d\left[\left(B_{1} t^{\alpha}+B_{2}\right) E_{\alpha}\left(\lambda t^{\alpha}\right)\right] . \\
c x=\Gamma(1+\alpha) B_{1} E_{\alpha}\left(\lambda t^{\alpha}\right)+(\lambda-d)\left[\left(B_{1} t^{\alpha}+B\right) E_{\alpha}\left(\lambda t^{\alpha}\right)\right], \\
x=\frac{1}{c}\left[\Gamma(1+\alpha) B_{1} E_{\alpha}\left(\lambda t^{\alpha}\right)+(\lambda-d)\left[\left(B_{1} t^{\alpha}+B_{2}\right) E_{\alpha}\left(\lambda t^{\alpha}\right)\right],\right. \\
=\frac{1}{c}\left[\Gamma(1+\alpha) B_{1} E_{\alpha}\left(\lambda t^{\alpha}\right)+(\lambda-d)\left[\left(B_{1} t^{\alpha} E_{\alpha}\left(\lambda t^{\alpha}\right)+(\lambda-d) B_{2} E_{\alpha}\left(\lambda t^{\alpha}\right)\right],\right.\right. \\
=\frac{1}{c}\left[( \lambda - d ) \left[\left(B_{1} t^{\alpha} E_{\alpha}\left(\lambda t^{\alpha}\right)+\left[\Gamma(1+\alpha) B_{1}+(\lambda-d) B_{2}\right] E_{\alpha}\left(\lambda t^{\alpha}\right)\right],\right.\right. \\
=\frac{1}{c}(\lambda-d)\left[\left(B_{1} t^{\alpha} E_{\alpha}\left(\lambda t^{\alpha}\right)+\left[\Gamma(1+\alpha) B_{1}+(\lambda-d) B_{2}\right] E_{\alpha}\left(\lambda t^{\alpha}\right)\right],\right. \\
=A_{1} t^{\alpha} E_{\alpha}\left(\lambda t^{\alpha}\right)+A_{2} E_{\alpha}\left(\lambda t^{\alpha}\right), \\
=\left(A_{1} t^{\alpha}+A_{2}\right) E_{\alpha}\left(\lambda t^{\alpha}\right),
\end{gathered}
$$

dimana $A_{1}=\frac{(\lambda-d) B_{1}}{c}, A_{2}=\frac{\Gamma(1+\alpha) B_{1}+(\lambda-d) B_{2}}{c}$.

Teorema 3.3. [2] Jika $(a-d)^{2}-4(a d-b c)<0$ dan $\lambda_{1}=p+i q, \lambda_{2}=p-i q$, maka solusi dari (3.1) adalah:

$$
\begin{aligned}
& x=E_{\alpha}\left(p t^{\alpha}\right)\left[M_{1} \cos _{\alpha}\left(b t^{\alpha}\right)+N_{!} \sin _{\alpha}\left(q t^{\alpha}\right)\right] \\
& y=E_{\alpha}\left(p t^{\alpha}\right)\left[M_{2} \cos _{\alpha}\left(q t^{\alpha}\right)+N_{2} \sin _{\alpha}\left(q t^{\alpha}\right)\right]
\end{aligned}
$$

dimana $M_{2}$ dan $N_{2}$ adalah konstanta sebarang dan:

$$
M_{1}=\left(\frac{p-d}{c}\right) M_{2}+\frac{q}{c} N_{2} \text {, dan } N_{1}=\left(\frac{p-d}{c}\right) N_{2}-\frac{q}{c} M_{2} .
$$

Bukti. Turunan fractional ke $\alpha$ dari persamaan kedua (3.16) adalah:

$$
\begin{aligned}
D^{\alpha} y= & p E_{\alpha}\left(p t^{\alpha}\right)\left[M_{2} \cos _{\alpha}\left(q t^{\alpha}\right)+N_{2} \sin _{\alpha}\left(q t^{\alpha}\right)\right], \\
& +E_{\alpha}\left(p t^{\alpha}\right)\left[-M_{2} q \sin _{\alpha}\left(q t^{\alpha}\right)+N_{2} q \cos _{\alpha}\left(q t^{\alpha}\right)\right] .
\end{aligned}
$$

Pengurangan (3.17) dengan py, memberikan:

$$
\begin{aligned}
D^{\alpha} y-p y= & p E_{\alpha}\left(p t^{\alpha}\right)\left[M_{2} \cos _{\alpha}\left(q t^{\alpha}\right)+N_{2} \sin _{\alpha}\left(q t^{\alpha}\right)\right] \\
& +E_{\alpha}\left(p t^{\alpha}\right)\left[-M_{2} q \sin _{\alpha}\left(q t^{\alpha}\right)+N_{2} q \cos _{\alpha}\left(q t^{\alpha}\right)\right] \\
& -p\left(E_{\alpha}\left(p t^{\alpha}\right)\left[M_{2} \cos _{\alpha}\left(q t^{\alpha}\right)+N_{2} \sin _{\alpha}\left(q t^{\alpha}\right)\right]\right) \\
= & E_{\alpha}\left(p t^{\alpha}\right)\left[-M_{2} q \sin _{\alpha}\left(q t^{\alpha}\right)+N_{2} q \cos _{\alpha}\left(q t^{\alpha}\right)\right] .
\end{aligned}
$$

Turunan ke $\alpha$ dari (3.18) adalah:

$$
\begin{aligned}
D^{\alpha}\left(D^{\alpha} y-p y\right)= & D^{\alpha}\left(E_{\alpha}\left(p t^{\alpha}\right)\left[-M_{2} q \sin _{\alpha}\left(q t^{\alpha}\right)+N_{2} q \cos _{\alpha}\left(q t^{\alpha}\right)\right]\right), \\
D^{2 \alpha} y-p D^{\alpha} y= & p E_{\alpha}\left(p t^{\alpha}\right)\left[-M_{2} q \sin _{\alpha}\left(q t^{\alpha}\right)+N_{2} q \cos _{\alpha}\left(q t^{\alpha}\right)\right] \\
& +E_{\alpha}\left(p t^{\alpha}\right)\left[-M_{2} q^{2} \cos _{\alpha}\left(q t^{\alpha}\right)-N_{2} q^{2} \sin _{\alpha}\left(q t^{\alpha}\right)\right] .
\end{aligned}
$$


Dengan mensubstitusi persamaan (3.18) dan persamaan (3.16) ke (3.19) diperoleh

$$
\left.D^{2 \alpha} y-p D^{\alpha} y=p\left(D^{\alpha} y-p y\right)\right)-q^{2} y \text {. }
$$

Persamaan (3.20) menjadi

$$
D^{2 \alpha} y-2 p D^{\alpha} y+\left(p^{2}+q^{2}\right) y=0 .
$$

Persamaan (3.21) menunjukkan bahwa persamaan kedua (3.3) adalah solusi dari persamaan (3.4). Untuk mendapatkan solusi $x$ dari persamaan (3.1), substitusi persamaan kedua dari (3.16) ke persamaan kedua dari (3.1), menghasilkan:

$$
\begin{aligned}
& D^{\alpha} y=c x+d y \\
& D^{\alpha}\left[E_{\alpha}\left(p t^{\alpha}\right)\left[M_{2} \cos _{\alpha}\left(q t^{\alpha}\right)+N_{2} \sin _{\alpha}\left(q t^{\alpha}\right)\right]=c x+d\left[E_{\alpha}\left(p t^{\alpha}\right)\left[M_{2} \cos _{\alpha}\left(q t^{\alpha}\right)+N_{2} \sin _{\alpha}\left(q t^{\alpha}\right)\right]\right.\right. \\
& \Longleftrightarrow p E_{\alpha}\left(p t^{\alpha}\right)\left[M_{2} \cos _{\alpha}\left(q t^{\alpha}\right)+N_{2} \sin _{\alpha}\left(q t^{\alpha}\right)\right]+E_{\alpha}\left(p t^{\alpha}\right)\left[-M_{2} q \sin _{\alpha}\left(q t^{\alpha}\right)+N_{2} q \cos _{\alpha}\left(q t^{\alpha}\right)\right]= \\
& c x+d\left[E_{\alpha}\left(p t^{\alpha}\right)\left[M_{2} \cos _{\alpha}\left(q t^{\alpha}+N_{2} \sin _{\alpha}\left(q t^{\alpha}\right)\right)\right]\right] \\
& \Longleftrightarrow c x=(p-d)\left[E_{\alpha}\left(p t^{\alpha}\right)\left[M_{2} \cos _{\alpha}\left(q t^{\alpha}\right)+N_{2} \sin _{\alpha}\left(q t^{\alpha}\right)\right]\right]+\left[E_{\alpha}\left(p t^{\alpha}\left[-M_{2} q \sin _{\alpha}\left(q t^{\alpha}\right)+N_{2} q \cos _{\alpha}\left(q t^{\alpha}\right)\right]\right]\right. \\
& \Longleftrightarrow x=\frac{1}{c}\left(( p - d ) \left(\left[E_{\alpha}\left(p t^{\alpha}\right)\left[M_{2} \cos _{\alpha}\left(q t^{\alpha}\right)+N_{2} \sin _{\alpha}\left(q t^{\alpha}\right)\right]\right]+\left[E_{\alpha}\left(p t^{\alpha}\left[-M_{2} q \sin _{\alpha}\left(q t^{\alpha}\right)+N_{2} q \cos _{\alpha}\left(q t^{\alpha}\right)\right]\right]\right)\right.\right. \\
& =\left[E_{\alpha}\left(p t^{\alpha}\right)\right]\left(\frac{(p-d)}{c}\left[M_{2} \cos _{\alpha}\left(q t^{\alpha}\right)+N_{2} \sin _{\alpha}\left(q t^{\alpha}\right)\right]+\frac{q}{c}\left[-M_{2} \sin _{\alpha}\left(q t^{\alpha}\right)+N_{2} \cos _{\alpha}\left(q t^{\alpha}\right)\right]\right), \\
& =\left[E_{\alpha}\left(p t^{\alpha}\right)\right]\left(\frac{(p-d)}{c}\left[M_{2} \cos _{\alpha}\left(q t^{\alpha}\right)+N_{2} \sin _{\alpha}\left(q t^{\alpha}\right)\right]+\frac{q}{c}\left[N_{2} \cos _{\alpha}\left(q t^{\alpha}\right)-M_{2} \sin _{\alpha}\left(q t^{\alpha}\right)\right]\right), \\
& =\left[E_{\alpha}\left(p t^{\alpha}\right)\right]\left[M_{1} \cos _{\alpha}\left(q t^{\alpha}\right)+N_{1} \sin _{\alpha}\left(q t^{\alpha}\right)\right] .
\end{aligned}
$$

dimana $M_{1}=\left(\frac{p-d}{c}\right) M_{2}+\frac{q}{c} N_{2}$ dan $N_{1}=\left(\frac{p-d}{c}\right) N_{2}-\frac{q}{c} M_{2}$.

\section{Kesimpulan}

Turunan tipe Jumarie adalah salah satu metode yang dapat menyelesaikan beberapa sistem persamaan diferensial fraksional linier dengan orde $\alpha$.

\section{Ucapan Terima kasih}

Terima kasih kepada Bapak Dr. Mahdhivan Syafwan, Ibu Monika Rianti Helmi, M.Si, dan Ibu Riri Lestari, M.Si yang telah memberikan kritik dan saran untuk penulisan artikel ini.

\section{Daftar Pustaka}

[1] Ghosh. U, Sengupta. S, et al. 2015. Analytic Solution of Linear Fractional Differential Equation With Jumarie Derivative in Term of Mittag-Leffler Function. American Journal of Mathematical Analysis, 3(2), 32 - 38, doi:10.12691/ajma$3-2-2$.

[2] Ghosh. U, Sarkar. S, and Das. S,. 2015. Solution of System of linear Fractional Differential Equation with derivative of Jumarie type . Americal Journal of Mathematical Analisis, 3(3): 72 - 84. doi:10.12691/ajma-3-3-3. 
[3] Jumarie. G. 2008. Faoriers Transformation of Fractional Order Via MittagLeffler Function and Modified Riemend-Lioville Derivatives. J. Appl. Math.Informatics. 26. $1101-1121$.

[4] Jumarie. G. 2006. Modified Riemann-Liouville Derivative and Fractional Taylor Series of Nondifferentialble Functional Further Results. Computer and Mathematics with Applications 51. 1367 - 1376.

[5] Milici. Constantin, Gheorghe. D, J. Tanreiro. 2019. Introduction to Fractional Differential Equations. Springer, Switzerland.

[6] Miller. Kenneth S. 1993. An Introduction to The Fractional Calculus and Fractional Differential Equation. Simultaneusly, Canada.

[7] Ortigueira, M. D. 2011. Fractional Calculus For Scientists and Engineers. Springer. New York.

[8] Spiegel, M. R. 2010. Theory and Problem of Advanced Calculus. McGraw-Hill International Book Company. New York. 